\title{
Hand-Held Gamma-Ray Imaging Sensors Using Room-Temperature 3-Dimensional Position- Sensitive Semiconductor Spectrometers
}

\author{
Zhong He, Carolyn Lehner, Feng Zhang, David K. Wehe, Glenn F. Knoll \\ James Berry, and Yanfeng Du* \\ Nuclear Engineering and Radiological Sciences Department \\ The University of Michigan \\ Ann Arbor, Michigan 48109-2104 \\ * Currently at GE Global Research Center \\ 1 Research Circle $-K W B 617$ \\ Niskayuna, NY 12309
}

\begin{abstract}
This paper demonstrates the capability of compact gamma-ray imaging devices using 3dimensional position sensitive CdZnTe semiconductor gamma-ray spectrometers, developed at the University of Michigan. A prototype imager was constructed and tested using two $1 \mathrm{~cm}$ cube 3dimensional position sensitive CdZnTe detectors. Energy resolutions of $1.5 \%$ FWHM for single pixel events at $662 \mathrm{keV}$ gamma-ray energy were obtained on both detectors, and an angular resolution of about $5^{\circ}$ FWHM was demonstrated. The capabilities of proposed devices, which can cover a wider energy range up to $2.6 \mathrm{MeV}$, are discussed.
\end{abstract}

\section{INTRODUCTION}

The events of September 11, 2001, have increased national priorities for the development and deployment of instruments that could enhance capabilities to detect and monitor various nuclear materials. Advanced gamma ray sensors can play a role in this effort, particularly if they provide spectroscopic and imaging capabilities. For many applications, operation at room temperature and the avoidance of a requirement to cool the device would also be a significant advantage for hand-held portable instruments.

Isotopes associated with plutonium and uranium emit gamma rays in the energy range from $186 \mathrm{keV}$ up to $2.6 \mathrm{MeV}$. Therefore, the measurement of characteristic gamma-ray lines can determine the presence of nuclear materials. Since the intensity of gamma rays from a point source is inversely proportional to the square of the distance to the source, hand-held gamma-ray sensors that enable inspection close to the source will increase the sensitivity of the measurement. In addition, if the instrument can provide the incident direction of gamma rays, gamma-ray spectroscopy can be performed for localized objects, thus further improving the sensitivity. In this paper, we investigate the performance of compact gamma-ray imaging sensors made using 3-dimensional position-sensitive CdZnTe gamma-ray spectrometers. These

CP632, Unattended Radiation Sensor Systems for Remote Applications, edited by J. I. Trombka et al.

$\odot 2002$ American Institute of Physics 0-7354-0087-3/02/\$19.00 
instruments allow room temperature operation, close-range inspection due to the compactness of the devices, and have imaging capability so that a source can be identified both by the spectroscopic measurement and by its location.

For systems in which small size and portability is important, the imaging method based on Compton scattering becomes a leading choice for gamma rays in the energy range between a few hundred $\mathrm{keV}$ to a few $\mathrm{MeV}$. This technique does not require a collimator, which makes the device light-weight; covers a wide angular field of view, making inspection more efficient, and does not require compromise between angular resolution and device sensitivity. This paper demonstrates the capability of compact gamma-ray imaging devices using 3-dimensional position sensitive $\mathrm{CdZnTe}$ semiconductor gamma-ray spectrometers, which are being developed at the University of Michigan.

\section{SYSTEM DESCRIPTION}

The principle of a Compton imaging device can be described as follows: If the energy depositions and 3-dimensional coordinates of each gamma-ray interaction can be recorded, the original gamma-ray energy and the incident angle $\varphi$ with respect to the axis defined by the first two interaction positions of the incident gamma ray can be obtained based on the Compton scattering formula:

$$
\cos \varphi=1-\frac{m_{e} c^{2}}{E_{\text {Scattering }}}+\frac{m_{e} c^{2}}{E_{\text {Incident }}}
$$

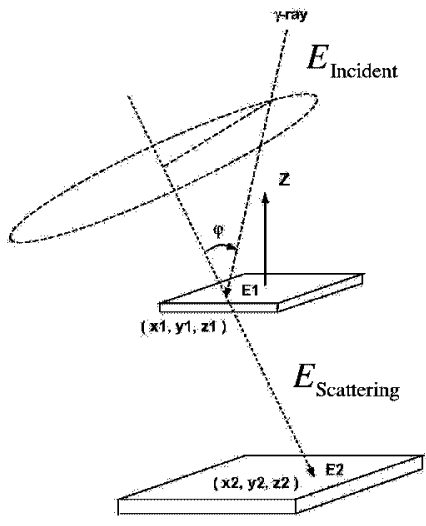

FIGURE 1. Illustration of a Compton-scattering imager.

In our first realization of the technique using $\mathrm{CdZnTe}$, we employed two separate detectors as indicated in Figure 1. Two $1 \times 1 \times 1 \mathrm{~cm}$ cubic CdZnTe crystals were located in close proximity, and each provided 3-D spectroscopic readout. Pixellated anodes on each detector were connected to VA1 ASIC electronics through wire bonds [1]. The operational mode was to select single events in each detector, for which the first is a Compton scatter and the second is a photoelectric absorption. The second generation 
detectors were fabricated during 2000 to 2001 using $1.5 \times 1.5 \times 1 \mathrm{~cm}$ CdZnTe crystals, and the connection between pixel anodes and the inputs of ASIC are realized using plate-through-via technique. This anode-ASIC connection is much more rugged than the wire bonding used on first generation detectors. In a 3-D spectroscopic detector of sufficient size, a single detector can replace the function of the two individual detectors shown in Figure 1. Multiple interactions of each entering gamma ray photon are now recorded, and the sequence of these interactions reconstructed to provide the same kind of information. We are currently working on a large-volume CdZnTe detector that will begin to offer attractive detection efficiencies in this mode. It is based on a crystal grown by Yinnel Tech Inc. that was $2 \times 2 \times 1.5 \mathrm{~cm}$ in dimension when received. After etching and polishing at $\mathrm{eV}$ Products facilities, the final dimension will be $\sim 1.8 \times 1.8 \times 1.3 \mathrm{~cm}$.

\section{ENERGY RESOLUTION}

The overall energy resolution of a detector system is determined by the energy resolution of each individual detector. Energy resolutions of about $1.5 \%$ were demonstrated on the first generation $1 \mathrm{~cm}^{3}$ detectors [1], and similar energy resolution is achieved on second generation detectors having $2 \mathrm{~cm}^{3}$ detection volume. An example is shown in Figure 2.

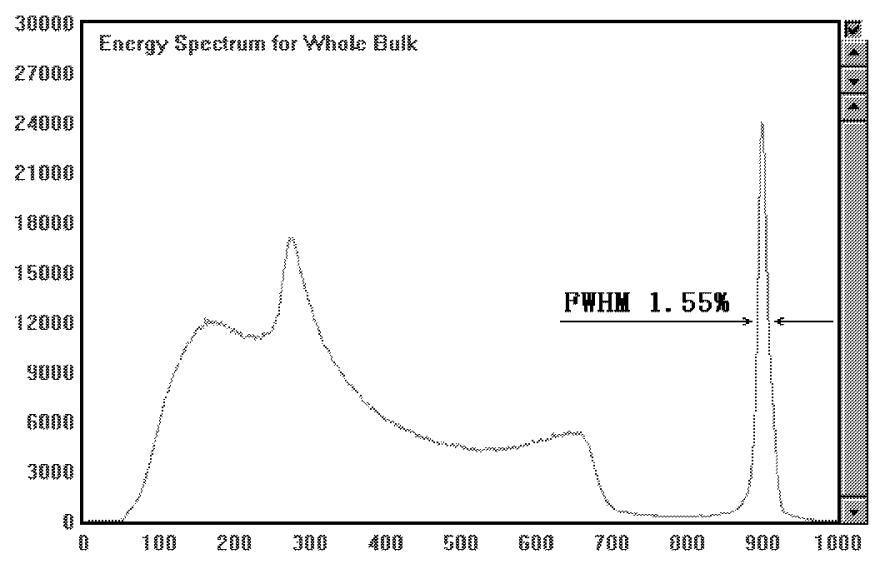

FIGURE 2. An energy spectrum of ${ }^{137} \mathrm{Cs}$ for single-pixel events obtained from a second generation 3dimensional position sensitive $\mathrm{CdZnTe}$ detector. The spectrum was obtained from all 108 working pixel anodes, with a cathode bias voltage of $-1000 \mathrm{~V}$. The detector dimensions are $1.5 \times 1.5 \times 1 \mathrm{~cm}$.

\section{ANGULAR RESOLUTION}

The angular resolution of a Compton scattering detector system is mainly limited by three factors: the detector energy resolution, position resolution (geometrical contribution) and the Doppler broadening effect [2]. Figure 3 shows the first prototype imaging system using two $1 \mathrm{~cm}^{3}$ 3-dimensional position sensitive $\mathrm{CdZnTe}$ 
spectrometers separated by a gap of $4 \mathrm{~cm}$. The angular uncertainty due to each factor discussed above was investigated, and is shown in Figure 4 at a gamma-ray energy of $1 \mathrm{MeV}$. Experimental measurements showed an angular resolution of about 5 degrees FWHM at $662 \mathrm{keV}$ gamma-ray energy, which is somewhat worse than 3.5 degrees predicted by Monte-Carlo simulations. It should be pointed out that the angular resolution improves at higher energies because of better energy resolution and the reduced effect of Doppler broadening on angular uncertainty.

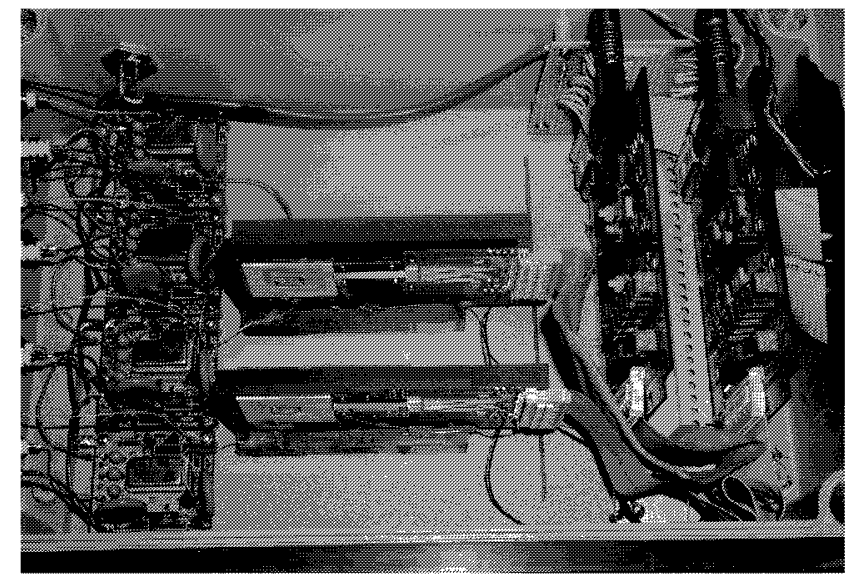

FIGURE 3. The top view of the first prototype Compton-scatter imaging system using two $1 \mathrm{~cm}$ cube 3-dimensional position-sensitive CdZnTe detectors.

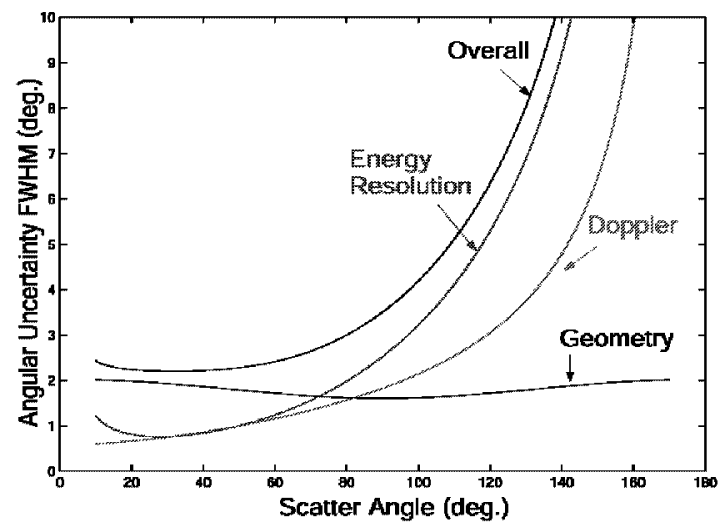

FIGURE 4. The angular resolution as a function of scattering angle for $1 \mathrm{MeV}$ gamma rays predicted by Monte-Carlo simulations. Consistent results were obtained from experimental tests. The contributions to angular uncertainty from energy resolution, position resolution and the Doppler effect are shown individually.

When the new $1.8 \times 1.8 \times 1.3 \mathrm{~cm} \mathrm{CdZnTe}$ detector is operated as an independent imager, the angular resolution will be dominated by geometrical uncertainty (position resolution). By assuming a position resolution of about $1.5 \mathrm{~mm}$ and an average separation of $6 \mathrm{~mm}$ (about half of the detector thickness) between two gamma-ray 
scattering locations, an angular resolution of about $\Delta \theta \approx 24^{\circ}$ FWHM should be expected (compared to $1 \mathrm{~mm}$ position resolution and $5 \mathrm{~cm}$ average separation between scatterings in the first prototype system). When a second detector is added to the system, and is separated from the first detector by $4 \mathrm{~cm}$, similar angular resolutions of $3-5^{\circ} \mathrm{FWHM}$ at $662 \mathrm{keV}$, and $2.5^{\circ}-4^{\circ} \mathrm{FWHM}$ at $1 \mathrm{MeV}$ should be achievable. The addition of a third detector should not change the angular resolution significantly, but would increase the detection efficiency at higher energies.

\section{DETECTION EFFICIENCY}

An important property of a detector system is its sensitivity, which is determined by detector area, photopeak efficiency and photopeak fraction. Although it is still difficult to obtain single crystal $\mathrm{CdZnTe}$ with volumes larger than a few $\mathrm{cm}^{3}$, the photopeak efficiency and in particular the photopeak fraction can be significantly improved by making intelligent use of the 3-D position resolution. An example is shown in Figure 5 at incident gamma-ray energy of $2.6 \mathrm{MeV}$, which are of interest in arms control applications. The energy spectra from a CdZnTe detector system with dimensions of $2 \times 2 \times 4.5 \mathrm{~cm}$, which could be made using three $2 \times 2 \times 1.5 \mathrm{~cm}$ detector modules similar to the one being constructed, were investigated using Monte-Carlo simulations. The energy spectrum when the detector is operated as a simple gamma-ray spectrometer is shown on the left of Figure 5. A photopeak efficiency of about $10 \%$ and a peak-tototal ratio (photopeak fraction) of about $16 \%$ were obtained. The single and double escape peaks are clearly visible from pair production events. In a real environment, characteristic gamma-ray lines are superimposed on a gamma-ray background from the Compton continuum and escape peaks of higher energy gamma rays. The suppression of this background can significantly increase the sensitivity of the detection system. By using a 3-dimensional position sensitive detector, the complex signature of radiation interactions with the detection medium can be recognized so that events of interest can be selected and others rejected to reduce the background noise. For example, some events in the single escape peak can be recognized if a $511 \mathrm{keV}$ energy deposition is detected at one location (through a photoelectric interaction) in coincidence with another energy deposition from the electron-positron pair generated in a pair production event. The correct photopeak energy can be obtained by adding $1022 \mathrm{keV}$ energy (of two $511 \mathrm{keV}$ gammas) to the energy deposition of the electronpositron pair. If there are three or more energy depositions detected in the system, the energy of the incident gamma-ray can be correctly reconstructed if the sequence of gamma-ray interaction can be recognized based on the kinematics of Compton scattering [3]. In addition, the majority of the Compton continuum and double escape events can be rejected if only a single energy deposition within the detector is recorded in the energy range for which the probability of a photoelectric interaction is very low. By implementing the event selection and reconstruction processes discussed above, the photopeak efficiency can be increased to about $15 \%$ (or improved by a factor of $\sim 50 \%$ ). Most importantly, the photopeak fraction is significantly increased to about 59\%. As can be seen in Figure 5, the Compton continuum is reduced significantly, especially below the energy of the double escape peak. It should be noted that further reduction of the continuum between the double escape energy and 
the photopeak is possible. Some of the $511 \mathrm{keV}$ gamma rays emitted from the annihilation of the electron-positron pair can be recognized if they deposit all their energy through Compton scattering in the device. This technique can be very useful if multiple gamma-ray lines must be observed, such as gamma rays at $2.6 \mathrm{MeV}, 1.001$ $\mathrm{MeV}$ and $911 \mathrm{keV}$ for the identification of highly enriched uranium. Because the background continuum from higher energy gamma rays will be significantly reduced, the signal to noise ratio will be improved and the effective volume of a 3-dimensional position sensitive detector system can be several times larger than that of a simple spectrometer having the same detection volume. So far, we have not discussed the improvement of the system sensitivity due to the imaging capability of the instrument. By observing gamma rays from localized directions, the natural background, which tends to come from all $4 \pi$ solid angles, can be further reduced.
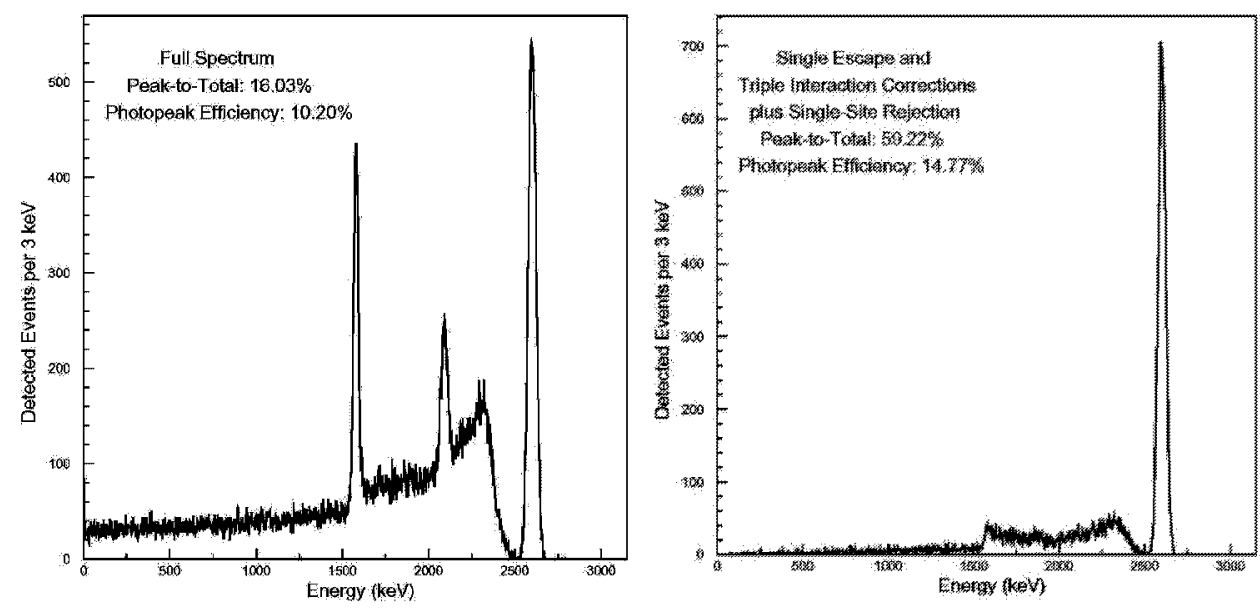

FIGURE 5. The comparison of energy spectra simulated from a $2 \times 2 \times 4.5 \mathrm{~cm}$ CdZnTe detector system, when the detector is operated as a simple spectrometer on the left, and as a 3-dimensional positionsensitive spectrometer on the right. $2.6 \mathrm{MeV}$ Gamma rays normally incident on the cathode surface in Monte-Carlo smulations.

\section{SUMMARY}

The performance of a compact Compton imaging system using modular 3dimensional position sensitive CdZnTe gamma-ray spectrometers has been investigated. Energy resolutions of about 1.5\% FWHM at $662 \mathrm{keV}$ gamma-ray energy have been demonstrated on prototype detectors, and angular resolutions of $\sim 24^{\circ}$ down to $3^{\circ}-5^{\circ} \mathrm{FWHM}$ at $662 \mathrm{keV}$ can be achieved depending on whether a single detector is employed, or 2 to 3 detector modules are used. A photopeak efficiency of $\sim 15 \%$ and a photopeak fraction of $\sim 59 \%$ can be achieved at $2.6 \mathrm{MeV}$, which should be of interest in nuclear non-proliferation applications. 


\section{ACKNOWLEDGMENTS}

We want to acknowledge the support of DOE NEER program, grant number DEFG07-98ID13645, and the support of DOE NN-20 office, grant number DE-FG0398NV13357.

\section{REFERENCES}

1. He Z. et al., Nuclear Instruments and Methods, A 422 (1999) 173-178.

2. Du Y.F., "Development of a Prototype Compton Scattering Camera using 3-D Position Sensitive CZT Detectors," Ph.D Thesis, The University of Michigan, 2001.

3. Dogan, N., "Multiple Compton Scatter Camera for Gamma Ray Imaging," Ph.D Thesis, The University of Michigan, 1993. 